\title{
Uniform Ergodicity of an Exponential Continuous Time $\operatorname{GARCH}(p, q)$ Model
}

\author{
Oesook Lee ${ }^{1, a}$ \\ ${ }^{a}$ Department of Statistics, Ewha Womans University
}

\begin{abstract}
The exponential continuous time $\operatorname{GARCH}(p, q)$ model for financial assets suggested by Haug and Czado (2007) is considered, where the log volatility process is driven by a general Lévy process and the price process is then obtained by using the same Lévy process as driving noise. Uniform ergodicity and $\beta$-mixing property of the log volatility process is obtained by adopting an extended generator and drift condition.
\end{abstract}

Keywords: Exponential continuous time $\operatorname{GARCH}(p, q)$ model, stationarity, uniform ergodicity, $\alpha$ mixing, $\beta$-mixing.

\section{Introduction}

Discrete time stochastic volatility models and GARCH processes which are capable of capturing some important stylized features such as jumps, heavy-tailedness, volatility clustering and dependence without correlation have been widely used in modeling financial volatility. Recently, however, financial data are treated mostly in continuous time. Continuous time processes are particularly appropriate for modeling irregularly-spaced and ultra high frequency data as they are useful in financial applications such as option pricing.

The continuous time $\operatorname{GARCH}(p, q)$ process is suggested by specifying the log-volatility process as the continuous time $\operatorname{ARMA}(q, p-1)$ process, which is the continuous time analogue of an $\operatorname{ARMA}(q, p-1)$ process (see Brockwell, 2001; Brockwell et al., 2006; Lindner, 2007).

Empirical observations show that stock returns are negatively correlated with changes in returns volatility. To represent this leverage effect, the following discrete time exponential $\operatorname{GARCH}(p, q)$ model is suggested by Nelson (1991);

$$
\begin{aligned}
Y_{n} & =\sigma_{n} e_{n}, \\
\log \left(\sigma_{n}^{2}\right) & =\mu+\sum_{k=1}^{p} \beta_{k} f\left(e_{n-k}\right)+\sum_{k=1}^{q} \alpha_{k} \log \left(\sigma_{n-k}^{2}\right) .
\end{aligned}
$$

Here, $f\left(e_{n}\right):=\theta e_{n}+\gamma\left[\left|e_{n}\right|-E\left(\left|e_{n}\right|\right)\right]$ with real coefficients $\theta$ and $\gamma, E\left|f\left(e_{1}\right)\right|<\infty$ and $\operatorname{Var}\left(f\left(e_{1}\right)\right)<\infty$, $\left(e_{n}\right)_{n \in Z}$ is a sequence of independent identically distributed random variables with $E\left(e_{1}\right)=0$ and $\operatorname{Var}\left(e_{1}\right)=1$. We also assume that $p, q \in N, \mu, \alpha_{1}, \ldots, \alpha_{q}, \beta_{1}, \ldots, \beta_{p}$ are constants in $R$ with $\alpha_{q} \neq 0$, $\beta_{p} \neq 0$ and that the autoregressive polynomial $\phi(z):=1-\alpha_{1} z-\cdots-\alpha_{q} z^{q}$ and the moving average polynomial $\psi(z):=\beta_{1}+\beta_{2} z+\cdots+\beta_{p} z^{q-1}$ have no common zeros and that $\phi(z) \neq 0$ on $\{z \in C \| z \mid \leq 1\}$. A

\footnotetext{
This research was supported by the Basic Science Research Program of the NRF funded by MEST (2010-0015707).

${ }^{1}$ Professor, Department of Statistics, Ewha Womans University, Daehyun-Dong Seodaemoon-Gu, Seoul 120-750, Korea.

E-mail: oslee@ewha.ac.kr
} 
continuous time version of the exponential $\operatorname{GARCH}(\operatorname{ECOGARCH})(p, q)$ model is proposed by Haug and Czado (2007), where the log volatility process is defined as the continuous time $\operatorname{ARMA}(q, p-1)$ process, and the stationarity, $\alpha$-mixing and moment properties of the process are investigated.

In this paper, we consider the $\operatorname{ECOGARCH}(p, q)$ model and prove the $V$-uniform ergodicity and $\beta$-mixing property of the log volatility process. $\alpha$-mixing property for a equidistance sequence of non-overlapping returns can be derived from $\beta$-mixing property of $\log$ volatility process.

\section{Preliminaries}

Let $L=\left(L_{t}\right)_{t \geq 0}$ be a time homogeneous càdlàg Lévy process with jumps $\Delta L_{t}=L_{t}-L_{t-}, t \geq 0$ defined on $(\Omega, \mathcal{F}, P)$ to $R$ starting from the origin. Denote by $\left(b, \tau^{2}, v\right)$ the characteristic triple of $L$. The Lévy measure $v$ is a nontrivial $\sigma$-finite measure on $R$ satisfying $v(\{0\})=0$ and $\int_{R} \min \left(1,|z|^{2}\right) v(d z)<\infty$.

For a Lévy process $L$ with zero mean, finite variance and nonzero parameters $\theta$ and $\gamma$, we define the driving process $M=\left(M_{t}\right)_{t \geq 0}$ of the log volatility process by

$$
M_{t}:=\int_{R-\{0\}} h(x) \tilde{N}_{L}(t, d x), \quad t \geq 0
$$

with $h(x):=\theta x+\gamma|x|$. Here $\tilde{N}_{L}(t, d x)$ is the compensated Poisson random measure defined by

$$
\tilde{N}_{L}(t, d x):=N_{L}(t, d x)-t v(d x)
$$

with $N_{L}(t, d x)$ as the associated Poisson random measure for $\Delta L$. The characteristic triple of $M$ is $\left(\gamma_{M}, 0, v_{M}\right)$ where $\gamma_{M}=-\int_{|x|>1} x v_{M}(d x), v_{M}=v \circ h^{-1}$ (see Applebaum, 2004, p.94).

The following $\operatorname{ECOGARCH}(p, q)$ process corresponding to the discrete time $\operatorname{EGARCH}(p, q)$ process of (1.1) is defined by specifying the $\log$-volatility process as a $\operatorname{CARMA}(q, p-1)$ process.

Definition 1. (Haug and Czado, 2007) Suppose that L has zero mean and finite variance. ECOGA $R C H(p, q)$ process $\left(G_{t}\right)_{t \geq 0}$ is defined by

$$
d G_{t}:=\sigma_{t-} d L_{t}, \quad t>0, G_{0}=0,
$$

where the log-volatility process $\log \left(\sigma_{t}^{2}\right)_{t \geq 0}$ is a CARMA(q,p-1) process, $1 \leq p \leq q$, with mean $\mu \in R$ and state space representation

$$
\begin{aligned}
\log \left(\sigma_{t}^{2}\right) & :=\mu+b^{T} X_{t}, \quad t>0, \quad \log \left(\sigma_{0}^{2}\right)=\mu+b^{T} X_{0}, \\
d X_{t} & =-A X_{t} d t+I_{q} d M_{t}, \quad t>0
\end{aligned}
$$

where $X_{0} \in R^{q}$ is independent of the driving Lévy process $M$ and $b^{T}$ is the transpose of $b$. The $q \times q$ matrix $A$, the vectors $b \in R^{q}$, and $I_{q} \in R^{q}$ are defined by

$$
A=\left(\begin{array}{ccccc}
0 & -1 & 0 & \cdots & 0 \\
0 & 0 & -1 & \cdots & 0 \\
\vdots & \vdots & \vdots & \ddots & \vdots \\
0 & 0 & 0 & \cdots & -1 \\
a_{q} & a_{q-1} & a_{q-2} & \cdots & a_{1}
\end{array}\right), \quad b=\left(\begin{array}{c}
b_{1} \\
b_{2} \\
\vdots \\
b_{q-1} \\
b_{q}
\end{array}\right), \quad I_{q}=\left(\begin{array}{c}
0 \\
0 \\
\vdots \\
0 \\
1
\end{array}\right)
$$

with coefficients $a_{1}, \ldots, a_{q}, b_{1}, \ldots, b_{q} \in R$, where $a_{q} \neq 0, b_{p} \neq 0$, and $b_{p+1}=\cdots=b_{q}=0$. 
The solution of (2.3) is given by

$$
X_{t}=e^{-A t} X_{0}+\int_{0}^{t} e^{-(t-s) A} I_{q} d M_{s}, \quad t>0 .
$$

Obviously, $X=\left(X_{t}\right)_{t \geq 0}$ in (2.4) is a Markov process whose sample path is càdlàg.

From now on, let $v_{M}$ denote the Lévy measure for the Lévy process $I_{q} M$ for notational simplicity. Writing $A=\left(a_{i j}\right)_{i, j=1}^{q}, x=\left(x_{1}, x_{2}, \ldots, x_{q}\right)^{T}, \partial_{j}=\partial / \partial x_{j}$, the infinitesimal generator $\mathcal{A}$ of $X$ is given by

$$
\mathcal{A} f(x)=-\sum_{i, j=1}^{q} a_{i j} x_{j} \partial_{i} f(x)+\gamma_{M} \partial_{q} f(x)+\int_{R^{q}}\left(f(x+z)-f(x)-\sum_{i=1}^{q} z_{i} \partial_{i} f(x) I_{|z| \leq 1}(z)\right) v_{M}(d z),
$$

since the Gaussian variance of the Lévy process $M$ is zero. $\mathcal{A}$ acts on the set of all real-valued $C^{2}\left(R^{q}\right)$ functions with compact support. $f \in C^{2}\left(R^{q}\right)$ implies that all the first and second partial derivatives of $f$ are continuous.

Let $\left(X_{t}\right)_{t \geq 0}$ be a continuous time Markov process with state space $R^{q}$ and transition probability function $P^{t}(x, A)=P\left(X_{t} \in A \mid X_{0}=x\right), x \in R^{q}, A \in \mathcal{B}\left(R^{q}\right)$.

A Markov process $\left(X_{t}\right)_{t \geq 0}$ is called $V$-uniformly ergodic, where $V \geq 1$ is a measurable function on $R^{q}$, if there exists a unique invariant measure $\pi$ for $P^{t}(\cdot, \cdot)$ such that

$$
\left\|P^{t}(x, \cdot)-\pi(\cdot)\right\|_{V} \leq V(x) d \rho^{t}, \quad t \geq 0, x \in R^{q}
$$

for some constants $d<\infty, 0<\rho<1$. Here the $V$-norm $\|\cdot\|_{V}$ is defined for any signed measure $\mu$ by $\|\mu\|_{V}:=\sup _{|g| \leq V}\left|\int g(y) \mu(d y)\right|$.

We denote by $D(\mathcal{A})$ the set of all functions $V: R^{q} \rightarrow R^{+}$for which there exists a measurable function $U: R^{q} \rightarrow R^{+}$such that, for each $x \in R^{q}, t>0$,

$$
\begin{aligned}
& E_{x}\left[V\left(X_{t}\right)\right]=V(x)+E_{x}\left[\int_{0}^{t} U\left(X_{s}\right) d s\right], \\
& \int_{0}^{t} E_{x}\left[\left|U\left(X_{s}\right)\right|\right] d s<\infty .
\end{aligned}
$$

We write $\mathcal{A} V:=U$ and call $\mathcal{A}$ the extended generator of the process $\left(X_{t}\right)_{t \geq 0}$.

For each positive integer $m$, let $O_{m}=\{x:|x|<m\}$ and $T^{m}=\inf \left\{t \geq 0:\left|X_{t}\right| \geq m\right\}$, i.e., the first entrance time to $O_{m}^{c}$. Define $X_{t}^{m}=X_{t} I_{\left\{t<T^{m}\right\}}+\Delta_{m} I_{\left\{t \geq T^{m}\right\}}$, where $\Delta_{m}$ is any fixed element in $O_{m}^{c}$. Define $\mathcal{A}_{m} V(x)=I_{O_{m}}(x) \cdot \mathcal{A} V(x)$.

A nonnegative measurable function $V \in D\left(\mathcal{A}_{m}\right)$ is called a norm-like function if $V(x) \rightarrow \infty$ as $x \rightarrow \infty$.

The following theorem plays a crucial role in proving the $V$-uniform ergodicity of a continuous time Markov process.

Theorem 1. (Theorem 6.1 in Meyn and Tweedie, 1993b) Suppose that $\left(X_{t}\right)_{t \geq 0}$ is a right process, and that all compact sets are petite for some skeleton chain. If there exist a norm-like function $V \geq 1$, constants $c>0$ and $d<\infty$, such that

$$
\mathcal{A}_{m} V(x) \leq-c V(x)+d, \quad x \in O_{m},
$$

then $\left(X_{t}\right)_{t \geq 0}$ is $V$-uniformly ergodic. 
Recall that $V$-uniformly ergodic processes are geometrically ergodic if $\int V(x) \pi(d x)<\infty$ for the invariant measure $\pi$, and the exponential convergence of (2.6) is equivalent to an exponential rate of mixing for the process.

Mixing properties play an important role in proving asymptotic results and are studied in literature, e.g., Doukhan (1994), Bradley (2005), Haug and Czado (2007), Masuda (2007) etc. For the process $\left(X_{t}\right)_{t \geq 0}$, we define $\mathcal{F}_{[s, t]}^{X}=\sigma\left(X_{u}: s \leq u \leq t\right)$ and

$$
\begin{aligned}
& \alpha_{X}(t)=\sup _{u \geq 0} \sup \left\{|P(A \cap B)-P(A) P(B)|: A \in \mathcal{F}_{[0, u]}^{X}, B \in \mathcal{F}_{[u+t, \infty)}^{X}\right\}, \\
& \beta_{X}(t)=\sup _{u \geq 0} E\left[\sup \left\{\left|P\left(B \mid \mathcal{F}_{[0, u]}^{X}\right)-P(B)\right|: B \in \mathcal{F}_{[u+t, \infty)}^{X}\right\}\right] .
\end{aligned}
$$

$X$ is called $\alpha$-mixing $\left(\beta\right.$-mixing) if $\alpha_{X}(t) \rightarrow 0\left(\beta_{X}(t) \rightarrow 0\right)$ as $t \rightarrow \infty$. If $\alpha_{X}(t) \leq K e^{-a t}\left(\beta_{X}(t) \leq K e^{-a t}\right)$ for some $a>0$ and $K>0$, then $X$ is called exponentially $\alpha$-mixing (exponentially $\beta$-mixing).

For detailed pertinent properties of Lévy processes, see Sato (1999) and Applebaum (2004). For terminologies and relevant results on Markov chain theory, we refer to Meyn and Tweedie (1993a, 1993b) and references therein.

\section{Uniform Ergodicity of $X_{t}$}

In this section we assume that the Lévy process $L$ has mean zero and finite variance and consider the processes $\sigma_{t}^{2}, \log \sigma_{t}^{2}, X_{t}$ and $G_{t}$ which are generated by equations (2.1)-(2.4). Given $X=\left(X_{t}\right)_{t \geq 0}$ is a time homogeneous Markov process whose sample path is càdlàg. Let $P^{t}(x, d y)$ be the probability transition function of $X$. Note that $X$ is a non-explosive Borel right process since $P^{t}$ maps Borel functions to Borel functions for each $t \geq 0$, where $\left(P^{t} f\right)(x):=\int f(y) P^{t}(x, d y)$.

Theorem 2. (Brockwell and Marquardt, 2005) If $X_{0}$ is independent of $\left\{I_{q} M_{t}, t \geq 0\right\}$, then $X$ is strictly stationary if and only if the eigenvalues of the matrix $A$ all have strictly positive real parts and $X_{0}$ has the distribution of $\int_{0}^{\infty} e^{-A s} I_{q} d M_{s}$. The strict stationarity of $X$ implies the strict stationarity of $\left(\sigma_{t}^{2}\right)_{t \geq 0}$ and $\left(\log \sigma_{t}^{2}\right)_{t \geq 0}$.

Following is our main theorem.

Theorem 3. Suppose that the eigenvalues of the matrix $A$ all have strictly positive real parts and that $\int_{|z|>1}|z|^{p} v_{M}(d z)<\infty$ for some $p>0$. Then $X$ is V-uniformly ergodic. If $X_{0}$ is independent of $\left\{I_{q} M_{t}, t \geq 0\right\}$ and $X_{0}$ has the distribution of $\int_{0}^{\infty} e^{-A s} I_{q} d M_{s}$, then $X$ is $\beta$-mixing with exponential decay rates.

Proof: Note that $X_{t}$ in (2.4) is a weak Feller process. Let $X^{(r)}=\left(X_{n}^{(r)}\right)_{n \in N_{0}}$ denote the discrete time Markov chain regularly sampled from $\left(X_{t}\right)_{t \geq 0}$ at the time points $0, r, 2 r, \ldots$ for a constant $r>0$. This $X^{(r)}$ is called the $r$-skeleton chain. Under the given assumptions, $P^{t}(x, A) \rightarrow \pi(A)$ as $t \rightarrow \infty$ for some limiting distribution $\pi$ (Masuda, 2004, Proposition 2.2) and hence the skeleton chain $X^{(r)}$ for some $r>0$ is irreducible and aperiodic. Therefore, every compact set is petite for $X^{(r)}$. In order to obtain the desired results we need to find a proper measurable function $V: R^{q} \rightarrow R^{+}$such that $V$ and $\mathcal{A}$ given in (2.5) satisfy the relations (2.7)-(2.9).

For some $p(0<p<1)$, we define $C^{2}$-function $V: R^{q} \rightarrow R^{+}$by $V(x)=|x|^{p}+1,|x|>1$ with continuous first and second partial derivatives on $|x| \leq 1$. 
Recall that the gradient vector of $f$ at $x$ is denoted by $\nabla f(x)$ and defined by the formula $\nabla f=$ $\left(\partial_{1} f, \partial_{2} f, \ldots, \partial_{q} f\right)$. For each $B$ bounded below, $N(t, B)$ denotes a Poisson random measure with intensity $v_{M}(B)$ and let $\tilde{N}(t, B):=N(t, B)-t v_{M}(B)$.

We may rewrite the equation (2.5) as

$$
\begin{aligned}
\mathcal{A} V(x)= & \nabla V(x)\left(-A x+I_{q} \gamma_{M}\right)+\int_{|z|>1}(V(x+z)-V(x)) v_{M}(d z) \\
& +\int_{|z| \leq 1}(V(x+z)-V(x)-\nabla V(x) \cdot z) v_{M}(d z) \\
= & \nabla V(x)\left(-A x+I_{q} \gamma_{M}\right)+I+I I .
\end{aligned}
$$

In this proof, we use $K<\infty$ as the universal constant and $K$ may vary from line to line. If $|x|>1$, then

$$
\begin{aligned}
|I| & =\left|\int_{|z|>1}(V(x+z)-V(x)) v_{M}(d z)\right| \\
& \leq \int_{|z|>1,|x+z|>1}|z|^{p} v_{M}(d z)+\int_{|z|>1,|x+z| \leq 1}|V(x+z)-V(x)| v_{M}(d z) \\
& \leq \int_{|z|>1}|z|^{p} v_{M}(d z)+\int_{|z|>1}\left(K+|z|^{p}\right) v_{M}(d z) \\
& \leq 2 \int_{|z|>1}|z|^{p} v_{M}(d z)+K v_{M}(|z|>1) \\
& <\infty
\end{aligned}
$$

The first inequality in (3.2) follows from ||$x+\left.z\right|^{p}-\left.|x|^{p}|\leq| z\right|^{p},(0<p \leq 1)$. Since for fixed $z,|z|>1$, $I_{|x+z| \leq 1}=0$ for $|x|>1+|z|$, the second inequality in (3.2) can be obtained.

For $|x| \leq 1$, we have that

$$
\begin{aligned}
|I| & =\int_{|z|>1,|x+z|>1}|x+z|^{p} v_{M}(d z)+K v_{M}(|z|>1) \\
& \leq \int_{|z|>1}|z|^{p} v_{M}(d z)+K v_{M}(|z|>1) .
\end{aligned}
$$

Now since $V(x+z)-V(x)-(z \cdot \nabla) V(x)=1 / 2(z \cdot \nabla)^{2} V\left(x_{\alpha}\right), x_{\alpha}=x+\alpha z, 0 \leq \alpha \leq 1$, by Lagrange remainder theorem,

$$
I I=\frac{1}{2} \int_{|z| \leq 1} \sum_{i=1}^{q} \sum_{j=1}^{q} z_{i} z_{j} \partial_{j} \partial_{i} V\left(x_{\alpha}\right) v_{M}(d z) .
$$

For the case that $|x| \leq 2$, we have that

$$
\begin{aligned}
|I I| & \leq \frac{1}{2} \int_{|z| \leq 1} \sum_{i=1}^{q} \sum_{j=1}^{q}\left|z_{i} z_{j} \partial_{j} \partial_{i} V\left(x_{\alpha}\right)\right| v_{M}(d z) \\
& \leq \sup _{\{|z| \leq 1,0 \leq \alpha \leq 1\}}\left\{\left|\partial_{j} \partial_{i} V\left(x_{\alpha}\right)\right|\right\} \int_{|z| \leq 1} \sum_{i=1}^{q} \sum_{j=1}^{q}\left|z_{i} z_{j}\right| v_{M}(d z)
\end{aligned}
$$




$$
\begin{aligned}
& \leq K \int_{|z| \leq 1}|z|^{2} v_{M}(d z) \\
& <\infty
\end{aligned}
$$

Boundedness of $\sup _{\{|z| \leq 1,0 \leq \alpha \leq 1\}}\left\{\left|\partial_{j} \partial_{i} V\left(x_{\alpha}\right)\right|\right\}$ follows from continuity of the second derivative of $V$. On the other hand, if $|x|>2$ and $|z| \leq 1$, then $|x+\alpha z| \geq 1$ and it can be derived by simple calculation that

$$
\partial_{j} \partial_{i} V\left(x_{\alpha}\right)= \begin{cases}p(p-2) x_{i} x_{j}|x+\alpha z|^{p-4}, & \text { if } i \neq j, \\ p|x|^{p-2}+p(p-2) x_{i}^{2}|x+\alpha z|^{p-4}, & \text { if } i=j\end{cases}
$$

and $\partial_{j} \partial_{i} V\left(x_{\alpha}\right)$ is bounded, since $0<p<1$. Therefore, we have that

$$
|I I| \leq K \int_{|z| \leq 1}|z|^{2} v_{M}(d z)<\infty
$$

Now, $\nabla V(x)=p|x|^{p-2} x^{T}$ for $|x|>1$ and $x^{T} A x \geq k|x|^{2}$ for some $k>0$ for all $x \in R^{q}$ imply that

$$
\begin{aligned}
\nabla V(x)\left(-A x+I_{q} \gamma_{M}\right) & =p|x|^{p-2}\left(-x^{T} A x+x^{T} I_{q} \gamma_{M}\right) \\
& \leq p|x|^{p-2}\left(-k|x|^{2}\right)+p|x|^{p-2} x^{T} I_{q} \gamma_{M} \\
& =-k p|x|^{p}+p \gamma_{M}|x|^{p-1} .
\end{aligned}
$$

From the definition of $V(X)$ and (3.6), $\nabla V(x)\left(-A x+I_{q} \gamma_{M}\right)$ is bounded on $|x| \leq 1$ and

$$
\nabla V(x)\left(-A x+I_{q} \gamma_{M}\right) \leq-k p V(x)+K, \quad|x|>1 .
$$

Combining (3.1)-(3.7) yields for some constants $c>0$ and $d>0$,

$$
\mathcal{A} V(x) \leq-c V(x)+d .
$$

Now, it remains to show that $V$ is a norm-like function, i.e., $V$ is in the domain of $\mathcal{A}_{m}$. Note that inequality in (3.8) implies that $E_{x}\left[V\left(X_{t}\right)\right] \leq e^{c t} V(x)$. Since $X_{t}$ is a finite variation process with right continuous paths and $V \in C^{2}$, Itô formula (see Protter, 2005, p.78) yields that

$$
\begin{aligned}
& V\left(X_{t}^{m}\right)-V\left(X_{0}^{m}\right) \\
& =\int_{0}^{t} \nabla V\left(X_{s-}^{m}\right) d X_{s}^{m}+\sum_{0<s \leq t}\left(V\left(X_{s}^{m}\right)-V\left(X_{s-}^{m}\right)-\nabla\left(X_{s-}^{m}\right) \Delta X_{s}^{m}\right) \\
& =\int_{0}^{t \wedge T^{m}} \nabla V\left(X_{s-}^{m}\right)\left(-A X_{s}+I_{q} \gamma_{M}\right) d s+\int_{0}^{t \wedge T^{m}} \nabla V\left(X_{s-}^{m}\right)\left(\int_{|z| \leq 1} z \tilde{N}(d s, d z)+\int_{|z|>1} z N(d s, d z)\right) \\
& \quad+\int_{R^{q}}\left(V\left(X_{s-}^{m}+z\right)-V\left(X_{s-}^{m}\right)-\nabla V\left(X_{s-}^{m}\right) z\right) \tilde{N}(t, d z)-\int_{0}^{t} \int_{R^{q}} \nabla V\left(X_{s-}^{m}\right) z I_{|z|>1} v_{M}(d z) d s \\
& =\int_{0}^{t \wedge T^{m}} \mathcal{A}_{m} V\left(X_{s-}^{m}\right) d s+\int_{0}^{t \wedge T^{m}} \int_{R^{q}} V\left(X_{s-}^{m}+z\right)-V\left(X_{s-}^{m}\right) \tilde{N}(d s, d z) .
\end{aligned}
$$

Now taking expectation on both sides of the equation (3.9), we obtain that

$$
E_{x}\left[V\left(X_{t}^{m}\right)\right]=V(x)+E_{x}\left[\int_{0}^{t} \mathcal{A}_{m} V\left(X_{s-}^{m}\right) d s\right] .
$$


(3.8) and (3.10) imply that $V(x)$ is a norm-like function satisfying the inequality (2.9). Hence by Theorem 1 (see also Theorem 5.2 in Down et al., 1995), $X$ is $V$-uniformly ergodic and $X$ with $\pi$ as its initial distribution is exponentially $\beta$-mixing. Moreover, $\int V(z) \pi(d z)<\infty$.

For self-completeness of this paper, we state the following theorem.

Theorem 4. Under the same assumption of Theorem 3, the following mixing properties hold; (1) $\left(\log \sigma_{t}^{2}\right)_{t \geq 0}$ and $\left(\sigma_{t}^{2}\right)_{t \geq 0}$ are exponentially $\alpha$-mixing. (2) The discrete time process $\left(G_{n r}^{(r)}\right)_{n \in N}$ where $G_{t}^{(r)}:=G_{t}-G_{t-r}=\int_{t-r}^{t} \sigma_{s-} d L_{s}, t>r>0$ is exponentially $\alpha$-mixing and ergodic.

Proof: Let for $X_{t}=\left(X_{t 1}, X_{t 2}, \ldots, X_{t q}\right)^{T}$, define that $Y_{t}=\left(Y_{t 1}, Y_{t 2}, \ldots, Y_{t q}\right)^{T}=B \cdot X_{t}$ where

$$
B=\left(\begin{array}{ccccc}
b_{1} & b_{2} & b_{3} & \cdots & b_{q} \\
0 & 1 & 0 & \cdots & 0 \\
\vdots & \vdots & \vdots & \ddots & \vdots \\
0 & 0 & \cdots & 1 & 0 \\
0 & 0 & 0 & \cdots & 1
\end{array}\right) .
$$

Then $Y_{t}=B \cdot X_{t}=\left(\log \sigma_{t}^{2}, X_{t 2}, \ldots, X_{t q}\right)^{T}$ and

$$
\begin{aligned}
Y_{t} & =B \cdot X_{t} \\
& =e^{-B A B^{-1} t} B X_{s}+\int_{s}^{t} e^{-B A B^{-1}(t-u)} B I_{q} d M_{u} .
\end{aligned}
$$

(1) If all eigenvalues of $A$ have positive real parts, so do all eigenvalues of $B A B^{-1}$. Therefore, applying Theorem 3 yields that $Y_{t}$ is $V$-uniformly ergodic for properly defined function $V$ and $\beta$-mixing. Since $\log \sigma_{t}^{2}$ is the first coordinate of $Y_{t}$, it is also $V$-uniformly ergodic and $\beta$-mixing. Mixing property of $\sigma_{t}^{2}$ follows from the fact that log function is continuous. (2) For details, see p.12 in Haug and Czado (2007).

\section{References}

Applebaum, D. (2004). Lévy Processes and Stochastic Calculus, Cambridge University Press.

Bradley, R. (2005). Basic properties of strong mixing conditions: A survey and some open questions, Probability Surveys, 2, 107-144.

Brockwell, P. J. (2001). Lévy driven CARMA process, Annals of the Institute of Statistical Mathematics, 53, 113-124.

Brockwell, P., Chadraa, E. and Lindner, A. (2006). Continuous time GARCH processes, Annals of Applied Probability, 16, 790-826.

Brockwell, P. and Marquardt, T. (2005). Lévy driven and Fractionally integrated ARMA processes with continuous time parameter, Statistica Sinica, 15, 477-494.

Doukhan, P. (1994). Mixing: Properties and Examples, Lecture Note in Statistics 85, Springer-Verlag, New York.

Down, D., Meyn, S. P. and Tweedie, R. L. (1995). Exponential and uniform ergodicity of Markov processes, The Annals of Probability, 23, 1671-1691.

Haug, S. and Czado, C. (2007). An exponential continuous time GARCH process, Journal of Applied Probability, 44, 960-976. 
Lindner, A. (2007). Continuous time GARCH processes, Handbook of Financial Mathematics, Sprin ger-Verlag.

Masuda, H. (2004). On multidimensional Ornstein-Uhlenbeck processes driven by a general Levy process, Bernoulli, 10, 97-120.

Masuda, H. (2007). Ergodicity and exponential $\beta$-mixing bounds for multidimensional diffusions with jumps, Stochastic Processes and Their Applications, 117, 35-56.

Meyn, S. P. and Tweedie, R. L. (1993a). Markov Chains and Stochastic Stability, Springer-Verlag, Berlin.

Meyn, S. P. and Tweedie, R. L. (1993b). Stability of Markovian processes III: Foster-Lyapunov criteria for continuous time processes, Advances in Applied Probability, 25, 518-548.

Nelson, D. B. (1991). Conditional heteroscedasticity in asset returns: A new approach, Econometrica, 59, 347-370.

Protter, P. E. (2005). Stochastic Integration and Differential Equations, 2nd Ed., Springer.

Sato, K. (1999). Lévy Processes and Infinitely Divisible Distributions, Cambridge University press, Cambridge. 